\title{
Flexible Bidentate Pyridyl Ligands in the Coordination-Driven Self-Assembly of Discrete Pd-Macrocycles
}

\author{
Mi-Eun Moon, Hyo Jin Lee, Bong Sung Ko, and Ki-Whan Chi \\ Department of Chemistry, Lniversity of Ulsan. L/san 680-749, Korea. "E-mail Lwchialsanackr \\ Recerved November 29, 2006
}

Key Words : Flexible pyridỵl ligands. Coordination-driven self-assembly . Pd-macrocycles

Pre-designed combination of bi- or tri-dentate pyridyl ligands and platinum or palladium containing acceptors has been used in recent years for the synthesis of discrete 2D or 3D supramolecules. ${ }^{\mathrm{j}}$ This coordination-driven self-assembly methodology has basically taken advantage of the fixed bonding directionality of ligands and acceptors to allow the prediction of assemibled product structures. ${ }^{2}$ Their fixed bond angles and the reversibility of dative bond formation have been ascribed to the unusual quantitative formation of discrete supramolecules instead of ufunite network. On the contrary, conformationally-flexible ligands have been rarely used for the self-assembly of discrete supramolecules. Template guest molecules or ions were required for the formation of discrete supramolecules self-assembled with flexible ligands. ${ }^{3}$ Only recently flexible ligands of 1,2-bis(3pyridyl)ethyne, 1.4-bis(3-pyridyl)-1.3-butadiyne and ambidentate pyridyl-carboxylate based donors have been successfully applied in the absence of template molecules for the coordination-driven self-assembly with various rigid platinum acceptors. ${ }^{+}$It is unique and interesting result that flexible ligands adjust their bond angle to acconmodate their rigid platinum acceptors for the self-assembly of discrete macrocycles. The right combination of a donor ligand and an acceptor metal as well as the degree of flexibility of ligands seen to be crucial for the successful coordination-driven self-assembly of discrete supramolecules. Thus, search for a new productive combination would be important work for expanding the applicability of coordinaton-driven selfassembly. On the other hand. like platimum analognes. square planar palladium complexes in which two positions are occupied by strong Pd-P bonds and remaining positions are coordinated by labile triflate anions have been popular acceptors in supramolecular chemistry. Therefore, we de- cided to check if flexible. bidentate pyridyl ligands like 1.2bis(3-pyridyl)etlyyne and 1.4-bis(3-pyridyl)-1.3-butadiyne would self-assemble with a rigid organopalladium acceptor into discrete supranolecular structures instead of oligomers or infinite networks. Herein, we report the results of our investigations.

The self-assembly processes were all perfonmed in the same general manner. $A C_{3} \mathrm{NO}_{2}(1.0 \mathrm{~mL})$ solution of ligands 1 ( $10 \mu \mathrm{mol}$ ) and organopalladinum 2 ( $10 \mu \mathrm{mol})$ was heated at $60^{\circ} \mathrm{C}$ for 3 hour, followed by evaporation of solvent with nitrogen gas to give a white solid. The solid product was washed with diethyl ether and dried under vacuum to provide the self-assembled product $3 \mathrm{in} \mathrm{high}$ yield. The formation of $[2+2\rceil$ supramolecules 3 is shown in Scheme 1. The self-assembled macrocycles were effectively characterized by ${ }^{2} \mathrm{P}\left\{{ }^{1} \mathrm{H}\right\}$ and ${ }^{1} \mathrm{H}$ NMR.

The ${ }^{21} \mathrm{P}\left\{{ }^{l} \mathrm{H}\right\}$ NMR spectra of 3 displayed a sharp singlet (3a: 68.37 ppm; 3b: 70.84 ppm). Sharp singlets are indicative of the formation of only one kind of product in each reaction. The signals are shifted approximately $8.3-10.8 \mathrm{ppm}$ upfield relative to $2(79.18 \mathrm{ppm})$ due to back donation from the palladinum centers (Figure 1). In the ${ }^{1} \mathrm{H}$ NMR spectra of 3 . the $\alpha$-hydrogen nuclei $\mathrm{H}_{\mathrm{a}}$ of the pyridine rings experienced downfield shifts $(0.2-0.8 \mathrm{ppm})$ relative to 1 . These are consistent with the loss of electron density upon coordination of the pyridine-N.

Further critical evidence of $\mathbf{3}$ was obtained with ESI-MS analysis. The self-assembled products 3 displayed similar spectra as shown in Figure 2 and 3. A peak corresponding to the intact macrocycle minus one $\mathrm{TfO}^{-}$counter ion was observed $\left\{\left[3 \mathrm{a}-\mathrm{TfO}^{-}\right]^{+}(m z \mathbf{z} 1817)\right.$. \{ $\left.\left[3 \mathrm{~b}-\mathrm{TfO}^{-}\right]^{+}(m z \mathbf{z} 1865)\right\}$. The second tallest peak was assigned to half of the intact cycle $\left\{\left[3 \mathrm{a} / 2-\mathrm{TfO}^{-}\right]^{-}(m z 833),\left\{\left[3 \mathrm{~b} / 2-\mathrm{TfO}^{-}\right]^{+}(m z 857)\right\}\right.$.
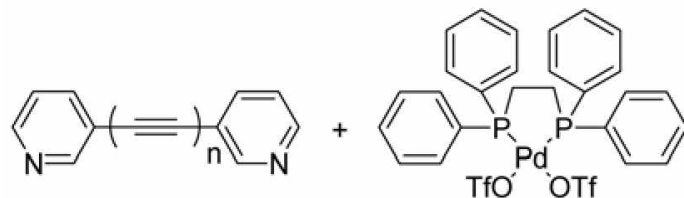

TfO'

1a: $n=1$

1b: $n=2$
2

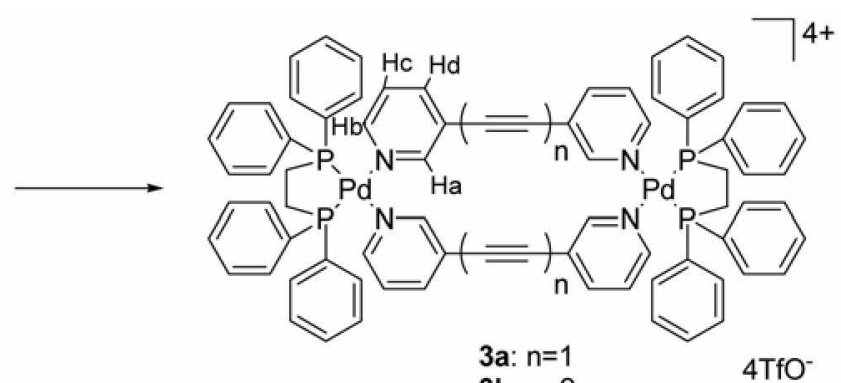

3b: $n=2$
$4 \mathrm{TfO}^{-}$

Scheme 1. Self-assembly of flexible, pyidyl ligands 1 with palladium acceptor 2. 


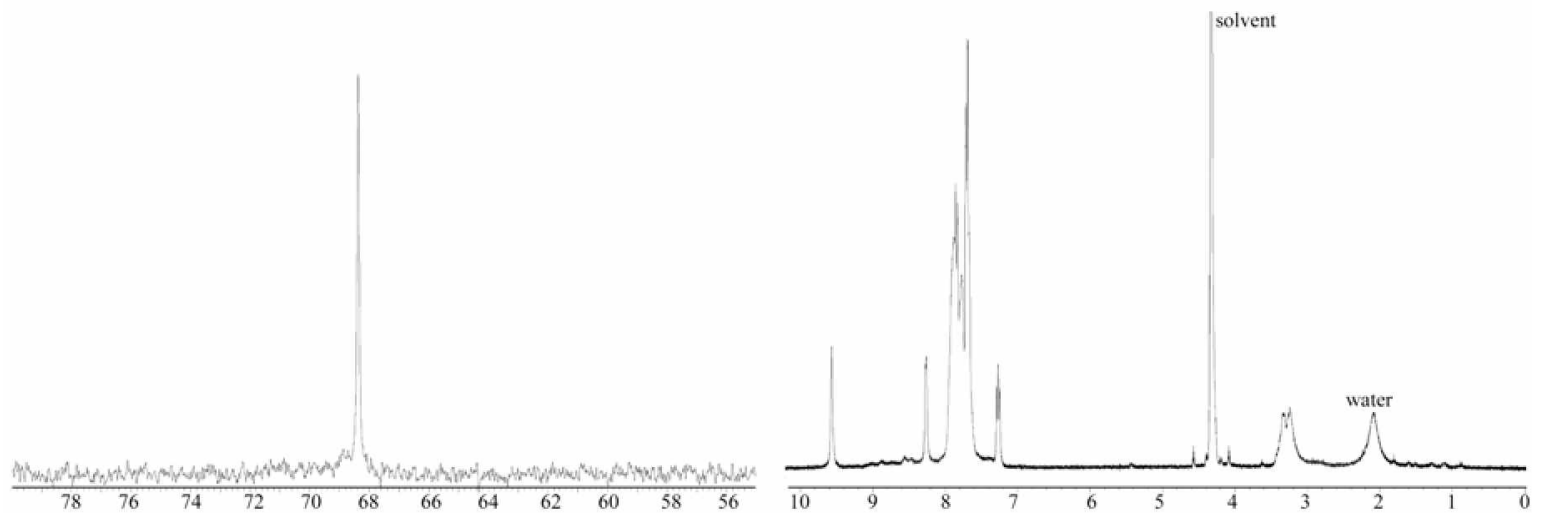

Figure 1. ${ }^{3} \mathrm{P}:{ }^{\mathrm{l}} \mathrm{H}$; and ${ }^{\mathrm{l}} \mathrm{H}$ NMR spectra of self-assembled macrocycle $3 a$.
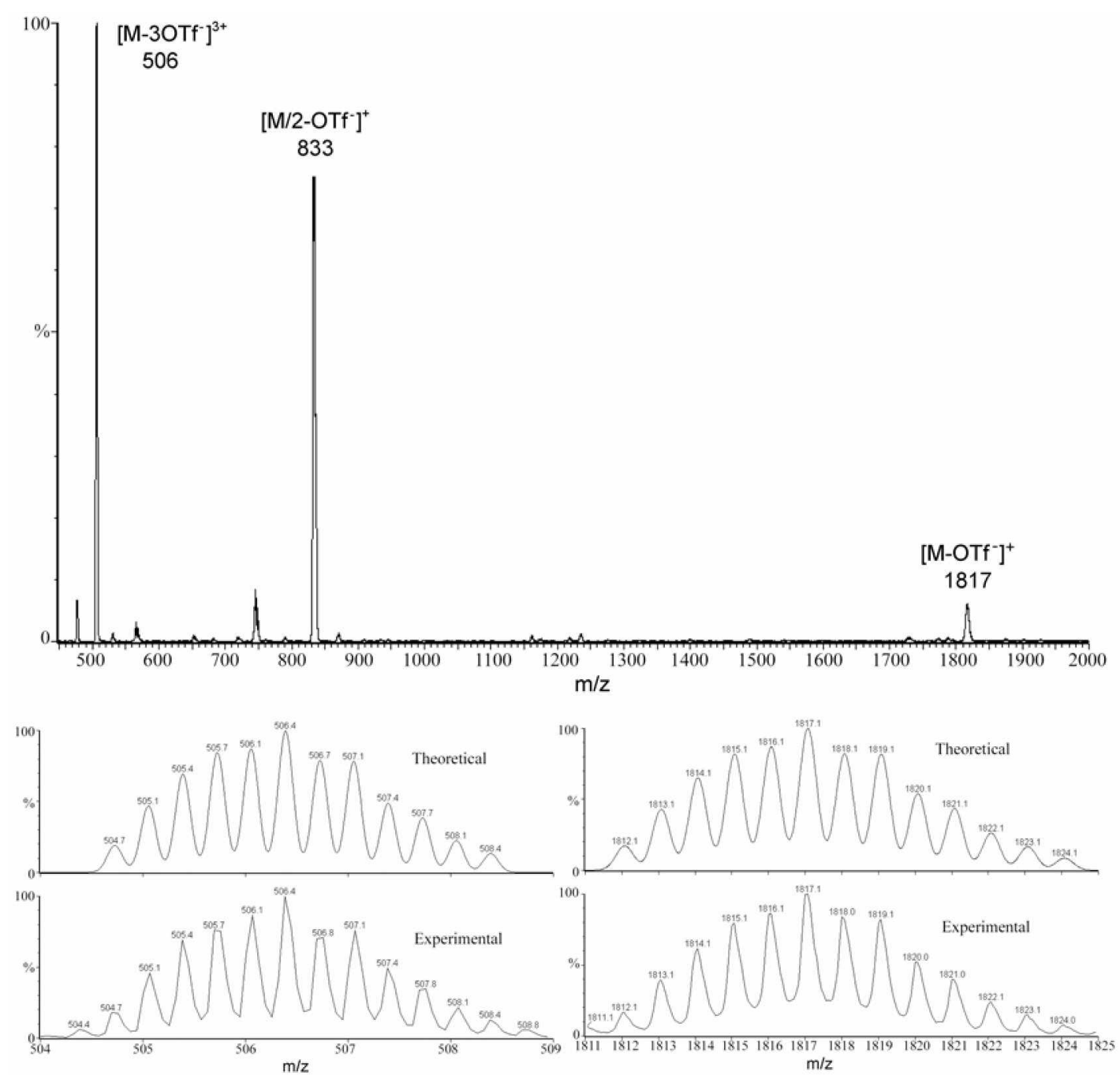

Figure 2. ESI-MS of 3a and the isotopically resolved peaks.

Presumably macrocycles 3 readily undergo fragmentation into half under the electron-spray analysis conditions employed. $^{\text {ta }}$ The base peak corresponding to the intact macrocycle ninus three $\mathrm{TfO}^{-}$ions was also observed $\left\{\left[3 \mathrm{a}-3 \mathrm{TfO}^{-}\right]^{3+}\right.$ $(m z 506)$. $\left\{\left[3 \mathrm{~b}-3 \mathrm{TfO}^{-}\right]^{3+}(m ; 222)\right\}$. These peaks were all isotopically resolved and match the theoretical distributions very well. And satisfactory elemental analysses of 3 were obtained

Both reactions provided one predominant species evident in the ${ }^{3 /} \mathrm{P}$ and ' $\mathrm{H}$ NMR spectra. Their sharp appearance suggests they are discrete species rather than oligomeric ones. In addition. no mass spectral evidence was found for any larger macrocycles. Impurity level in NMR spectra was smaller in the reaction with shorter ligand $\mathbf{l} \mathbf{a}$ than that of $\mathbf{1 b}$. 

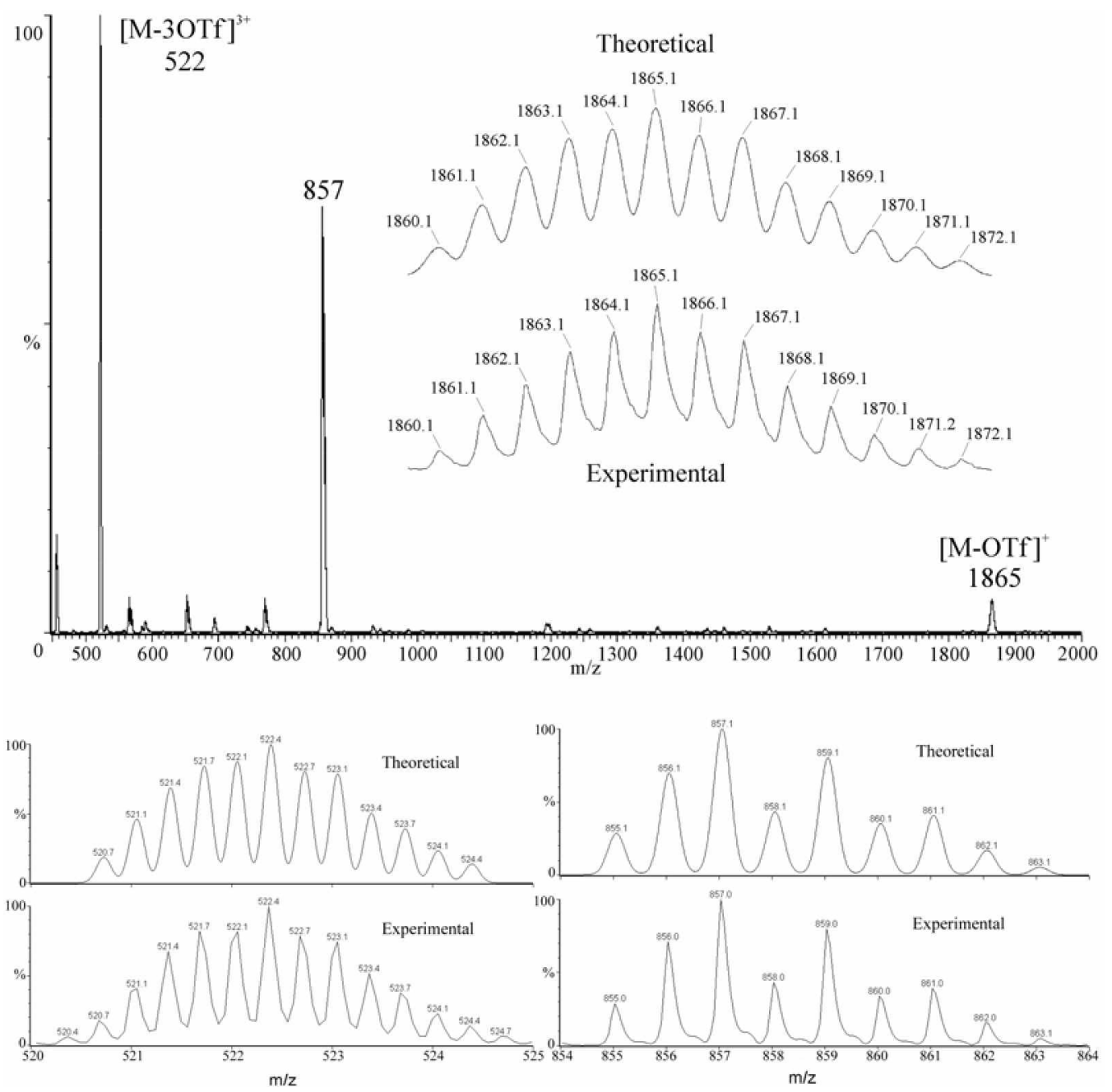

Figure 3. ESI-MS of 3b and the isotopically resolved peaks.

which reflects a general trend that less flexible ligands produce higher yields of specific self-assembled products.

In conclusion. we have prepared discrete macrocycles 3 from flexible. bidentate pyridyl ligands 1 and palladium containing acceptor 2 . Despite their ability to vary their bonding directionality. ligands 1 prefer to self-assemble into $[2+2]$ macrocycles by adjusting their bonding directionality to accommodate rigid organopalladium acceptor 2 . They represent unique examples of discrete supramolecules selfassembled from flexible pyrridyl-ligands and a rigid $\mathrm{Pd}$ acceptor in the absence of template guest molecules. This provides additional evidence of thermodynamic control favoring formation of discrete entities over oligomeric networks. presumably due to the added gain in enthalpy from the additional dative $\mathrm{Pd}-\mathrm{N}$ bond in the closed system. These molecular architectures represent further characteristic members in the self-assembled discrete supramolecules. illustrating the versatility of coordination-driven approach.

\section{Experimental}

Methods and Materials. The pyridyl ligands $1^{\circ}$ and organopalladiun compound $\mathbf{2}^{7}$ were prepared as reported.
All NMR spectra recorded on Varian Unity 300 spectrometer. ${ }^{1} \mathrm{H}$ and ${ }^{31} \mathrm{P}\{\mathrm{IH}\}$ chemical shifts are reported relative to the residual protons of $\mathrm{CD}_{3} \mathrm{NO}_{2}$ and an external, unlocked sample of $\mathrm{H}_{3} \mathrm{PO}_{4}(0.00 \mathrm{ppm})$ respectively. ESI mass spectra were recorded on a Micromass Quatto II Triple Quadrupole mass spectrometer with Micromass MassLỵnx operating sy'stem.

General Procedure for the Preparation of Assemblies 3. Organopalladium acceptor $2(10 \mathrm{cmol})$ and bidentate ligand 1 (10 $\mu \mathrm{mol})$ were placed in a l-dram vial. Nitromethane- $d_{3}(1.0 \mathrm{~mL})$ was added and the reaction was heated at $60^{\circ} \mathrm{C}$ for 3 hours. Solvents were evaporated by blowing with nitrogen gas and the remaining solid products were washed with diethyl ether. The self-assembled products 3 were finally dried under vacuum.

3a: Yield $96 \% . \mathrm{Mp}=218-222^{\circ} \mathrm{C}$ dec. ${ }^{1} \mathrm{H}$ NMR $\left(\mathrm{CD}_{2} \mathrm{NO}_{2}\right.$. $300 \mathrm{MHz}$ ) $\delta 9.57$ (s. $4 \mathrm{H} . \mathrm{H}_{a}$ ), 8.27 (br d, ${ }^{3} J=4.3 \mathrm{~Hz}, 4 \mathrm{H}$, $\mathrm{H}_{\mathrm{b}}$ ). $7.95-7.62$ (m. $40 \mathrm{H} . \mathrm{H}_{\mathrm{Ph}}$ ), 7.77 (br s. $\left.4 \mathrm{H}, \mathrm{H}_{\mathrm{d}}\right) .7 .27\left(\mathrm{t},{ }^{3} \mathrm{~J}\right.$ $\left.=6.9 \mathrm{~Hz} .4 \mathrm{H}, \mathrm{H}_{c}\right), 3.33\left(\mathrm{~m} .4 \mathrm{H}, \mathrm{PCH}_{2}\right), 3.24\left(\mathrm{~m}_{3}, 4 \mathrm{H} . \mathrm{CH}_{2} \mathrm{P}\right)$. ${ }^{31} \mathrm{P}\left\{{ }^{\prime} \mathrm{H}\right\}$ NMR $\left(\mathrm{CD}_{2} \mathrm{NO}_{2} .12 \mathrm{l} \mathrm{MHz}\right) \delta 68.37$ (s). Anal. Calcd. for $\mathrm{C}_{84} \mathrm{H}_{64} \mathrm{~F}_{1} \mathrm{~N}_{4} \mathrm{O}_{1} \geq \mathrm{P}_{4} \mathrm{Pd}_{4} \mathrm{~S}_{4} \cdot 2 \mathrm{H}_{2} \mathrm{O}$ : C. 47.99: H. 3.42; $\mathrm{N}$. 2.80. Found: C. 47.87; H. 3.23: N, 3.07 .

$3 \mathbf{b}$ : Yield $98 \% . \mathrm{Mp}>250^{\circ} \mathrm{C}$ dec. ${ }^{1} \mathrm{H}$ NMR $\left(\mathrm{CD}_{2} \mathrm{NO}_{2}, 300\right.$ 
$\mathrm{MHz}) \delta 8.97\left(\mathrm{~s}, 4 \mathrm{H}, \mathrm{H}_{3}\right) .8 .37$ (br s, $\left.4 \mathrm{H}, \mathrm{H}_{\mathrm{b}}\right), 7.94-7.62(\mathrm{~m}$. $\left.40 \mathrm{H}, \mathrm{H}_{\mathrm{Ph}}\right) .7 .69$ (br s, $\left.4 \mathrm{H} . \mathrm{H}_{\mathrm{s}}\right) .7 .34$ (br s. $4 \mathrm{H} . \mathrm{H}_{\mathrm{c}}$ ). 3.23 (m. $\left.4 \mathrm{H}, \mathrm{PCH}_{2}\right) .3 .15$ (m. $\left.4 \mathrm{H} . \mathrm{CH}_{2} \mathrm{P}\right):{ }^{31} \mathrm{P}\left\{{ }^{1} \mathrm{H}\right\}$ NMR $\left(\mathrm{CD}_{3} \mathrm{NO}_{2}\right.$. $121 \mathrm{MHz}) \delta 70.84$ (s). Anal. Calcd. for $\mathrm{C}_{84} \mathrm{H}_{64} \mathrm{~F}_{12} \mathrm{~N}_{4} \mathrm{O}_{1=-}$ $\mathrm{P}_{4} \mathrm{Pd}_{2} \mathrm{~S}_{4} \cdot 2 \mathrm{H}_{2} \mathrm{O}: \mathrm{C}, 49.20 ;$ H. 3.34: N. 2.73. Found: C. 49.01: H. 3.27: N. 2.88 .

Acknowledgement. This work was financially supported by the University of Ulsan Research Fund 2006.

\section{References}

1. (a) Yang. H.-B.: Das. N.: Huang. F.: Hawkridge. A. M.: Muddimant. D. C.: Stang. P. J J. Am. Chem. Soc. 2006. 128. 10014. (b) Fujita. M.: Tominaga. M: Hori, A.: Therrien. B. Acc. Them. Res. 2005. 38, 371. (c) Das, N.: Ghosh. A.: Arif. A. M.: Stang. P. J. horg. Chem. 2005, H. 7130 . (d) Mukherjee. P. S.: Das. N.: Kryschenko. Y. K.: Arif. A. M.: Stang. P. J. J. Am Chem. Soc. 2004. 126. 2464. (e) Seidel. S. R.: Stang. P. J. Acc. Chent. Res. 2002. 35.972. (f) Lehn. J.-M. Pros. Natl Acad Soi LS.4. 2002. 99. 4763. (g) Holliday. B. J.: Mirkin, C. A. Angew: them. Int Ed 2001, to. 2022. (i) Cotton, F. A.: Lin. C.: Murillo C. A Acc. Chem. Res. 2001, 34. 759. (i) Leininger. S.: Olenyluk. B.: Stang. P. J. Chem. Rev 2000. 100.853. (j) Caulder. D. L.: Raymond. K. N. J. Chem. Soc. Dalon Trans. 1999. 1185. (k) Caulder. D. L.: Raymond. K. N. Acc. Chem. Res. 1999. 32. 975. (1) Fujita. M. Chem. Soc. Rev: 1998. 6.417. (m) Stang. P. J.: Olenyuk. B. Acc. Chem. Res. 1997. 30,502

2. (a) Lehn. J.-M. Supramolecular Chemishy: Concepts and Perspectives: VCH: New York. 1995. (b) Chambron, J.-C.: Dietrich-
Buchecker. C.: Sauvage. J.P. In Compwhensive Supramolecthr Chentistry. Leht1. T.-M.. Chair. E.. Atwood. J. L.. Davis. T. E. D.. MacNico. D. D.. Vogtle. F.. Eds.: Pergamon Press: Osford. 1996: Vol. 9. Chapter 2. p 43. (c) Uller. E.: Demleitner. I.: Bernt. I.: Saalfrank, R. W. Symergistic Effect of Serendipity and Rational Design in Supramolecular Chemisty In Stuctwe and Bonding. Fujita. M.. Ed.: Springer: Berlin. 2000\%: Vol 96. p 149.

3. (a) Ikeda. A.: Udzu. H.: Zhong. Z.: Shitnkai. S.: Sakamoto. S.: Yamaguchi. K. J. An Chem. Soc. 2001. 123. 3872. (b) Liu. H.-K.: Sut1. W.-Y: Ma. D.-J:: Yu. K.-B.: Tang. W.X. Chent Conmum. 2000. 591. (c) Hiraoka S.: Kubota, Y.: Fujita, M. Chem. Commum. 2000. 1509. (d) Hiraoka. S.: Fujita, M. J. Am. Chem. Soc. 1999 121. 10239. (e) Fujita. M.; Nagao, S.: Ogura. K. J. Am. Chem. Soc. 1995. 117.1649 .

4. (a) Chi. K.W.: Addicott. C.: Moon. M.E.: Lee. H. J.: Yoon. S. C.: Stang. P. T. J. Org. Chem. 2006. 7l. 6662. (b) Chi. K.-W: Addicott, C. Arif, A. M: Stang. P. J. d. Am. Chem. Soc, 2004, 126. 16569. (c) Chi. K.W. Addicott C: Kryschento, Y. K: Stang. P. J. J. Org. Chem 2004. 69. 964. (d) Chi, K.W. Addicott C.: Arif. A. M.: Das. N.: Stang. P. T. J. Org. Chent 2003. 68.9798.

5. (a) Lee. S. T.: Kiml. J. S.: Lin. W. Inorg Chent 2004. 13.6579. (b) Zhong. Z.: Ikeda. A.: Shinkai. S. Sakamoto. S.: Yamaguchi. K. Org. Lett. 2001. 3, 1085. (c) Zhuravel. M; Grewal. N. S.: Glueck. D. S. Organometallics $2000,19,2882$

6. (a) Champness. N. R: Khlobystov. A. N: Majuga, A. G.: Schroder. M.: Zyk. N. V. Tetrahedron Lett. 1999. 40. 5413. (b) Rodriguez. J. G.: Martin-Villamil. R.: Can1o. F. H.: Fonseca. I. J. Chent Soc. Perkin Trams. 1 1997. 709

7. (a) Zhong, Z; Ikeda, A.: Ayabe. M; Shinkai, S.: Sakamoto, S.: Yamaguchi. K. J. Org. Chem 2001. 66, 10012. (b) Stang. P. J. Cao. D. H.: Saito. S.: Arif, A. M. J. Am. Chem. Soc. 1995. 117 . 6273 